\title{
Rotating flow within a droplet actuated with AC EWOD
}

\author{
R. Malk ${ }^{\mathrm{a}, *}$, Y. Fouillet ${ }^{\mathrm{a}}$, L. Davoust $^{\mathrm{b}}$ \\ a CEA, LETI, MINATEC, F-38054 Grenoble, France \\ ${ }^{\mathrm{b}}$ CNRS - LEGI, Microfluidics, Interfaces E Particles Group, BP 53, 38041 Grenoble Cedex 9, France
}

\begin{abstract}
Electrowetting-on-dielectric (EWOD) is now used in numerous microsystems like digital lab-on-chips. This paper deals with a characteristic hydrodynamic flow appearing in droplets actuated by EWOD with AC voltage. In the coplanar electrode configuration, two pairs of vortex flows are observed to form in a droplet centred on the electrode gap. All experiments are performed in oil as ambient phase and flows in the droplet are analysed using fluorescent beads. At the same time, droplet oscillations induced by AC EWOD are also revealed under stroboscopic lighting. These experiments show that vortex location can be controlled by frequency actuation with fair degree of reproducibility.
\end{abstract}

\section{Introduction}

Digital lab-on-chips (DLC) are an alternative to conventional lab-on-chips based on continuous flow within microchannels. DLC consists in handling discrete amounts of liquid all over a surface. The droplets are considered as micro reactors inside which biological (or chemical) reactions can be individually achieved. Different physical mechanisms have thus been studied to develop processes at a scale of a droplet including thermocapillarity [1], surface acoustic waves (SAW), dielectrophoresis, and asymmetric surface functionalisation [2].

Among these mechanisms, electrowetting-on-dielectric is now a well-known technique in DLC. This phenomenon represents a very promising way of handling small volumes of liquid in microsystems, with both low power consumption and high reliability. By electrically controlling the effective wettability of a droplet, liquid handling is made possible without the need for moving parts or external devices. Basic operations such as drop displacement, drop mixing, drop coalescence or dispensing are fully achieved in DLC (Fig. 1). As a consequence, EWOD actuation enables the integration of bio-protocols in a chip [3]. Immunoassay and PCR have been validated within an EWOD chip in [4], sample preparation for proteomics analysis with matrix-assisted laser desorption/ionisation mass spectrometer and DNA reparation are reported in [5] and [6], respectively.

\footnotetext{
* Corresponding author. Tel.: +33438780193.

E-mail address: rachid.malk@cea.fr (R. Malk).
}

Technology developments, especially the ones devoted to hydrophobic layers and the study of wetting behaviour under electric field, are certainly the key points of such lab-on-chips. Furthermore, other basic functions or applications within a DLC remain to be developed provided some EWOD associated phenomena are mastered. To that end, further investigations on EWOD are necessary. This paper reports on the experimental investigation of flows induced in a droplet actuated with AC EWOD in open configuration which is better adapted to fundamental studies of EWOD behaviours. The ultimate goal of this study is the monitoring of EWOD driven hydrodynamics at the scale of a drop with the long-term application of biological sample preparation.

\section{EWOD driven flows}

Hydrodynamic flows in a droplet using AC voltage have recently been observed by Ko et al. [7] from the 'classical' configuration (Fig. 2(a)) with air as ambient phase. The flow was observed using a laser sheet and fluorescent tracers, and a toroidal vortex flow was reported. Furthermore, droplet oscillations were simultaneously observed and the authors explained that the toroidal flow originates from such oscillations. However, the mechanisms of EWOD-induced flows still remain a subject of debate as demonstrated by our experiments performed on a different electrode configuration.

The configuration used in this paper is the 'coplanar electrode configuration' (Fig. 2(b)). In this configuration, the droplet spreads over two electrodes covered by insulating and hydrophobic layers. The equilibrium state is reached when the drop straddles both electrodes [8]. A major advantage of coplanar electrodes is that the 

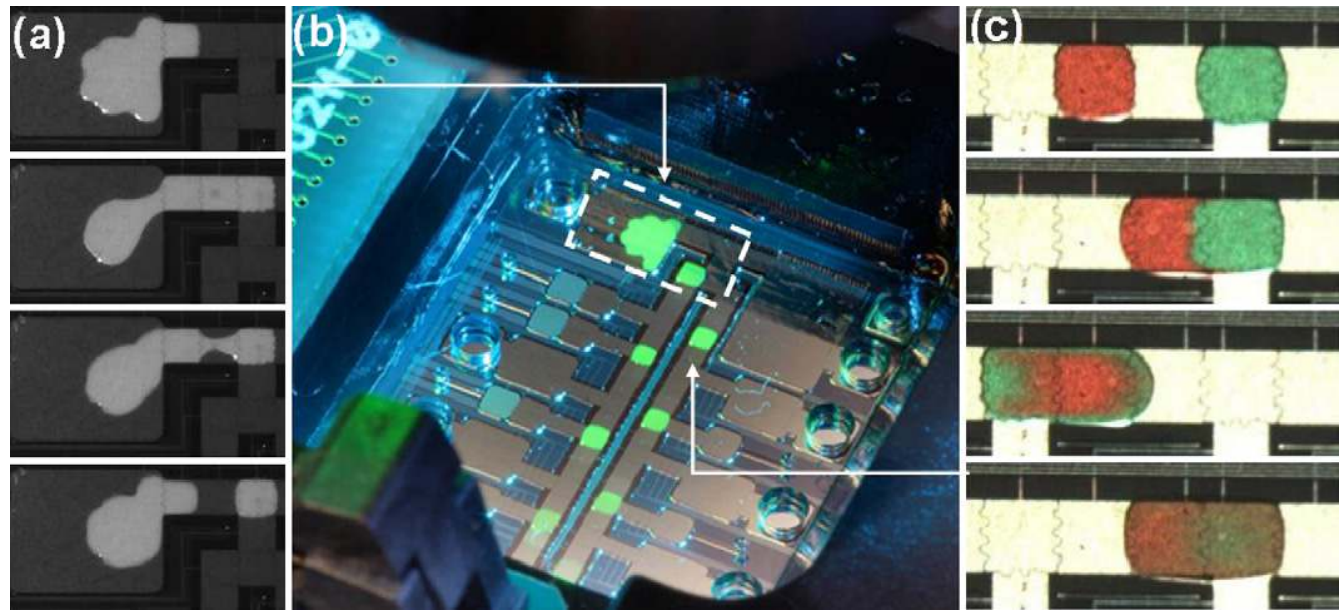

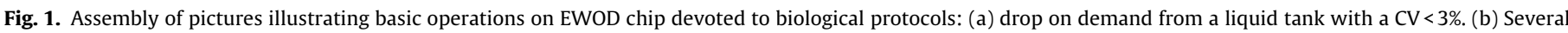
droplets are displaced from one electrode to the other. (c) Mixing with two colored droplets merged and moved back and forth.

droplet flow (and oscillations) is (are) not disturbed by any needle counter-electrode [9]. Furthermore, the coplanar configuration is generally more conventional for lab-on-chip in open configuration [10].

In these experiments, silicone oil was also used as ambient phase to prevent the droplet from evaporating. The droplet volume is fixed to $1.5 \mu \mathrm{l}$ with always the same aqueous buffer.

An optical setup has been developed in order to observe EWOD phenomena. It also enables the characterization of the wetting angle of a sessile drop over our configuration.

Vortex flows appearing on the droplet are analysed in this paper. Quadripolar vortices are clearly revealed. Numerous experiments were performed with different droplets and flow structures clearly appear to be very reproducible. Furthermore, vortex position on the drop surface is found to be directly controllable by the frequency of the applied voltage which, to the authors' knowledge, has never been reported before. To complement the digital toolbox based on EWOD microfluidics, such a flow could be considered as a means of developing new basic operations. So far, hydrodynamic flows within a droplet have been used for enhancing mixing [11,12], but the ability to control the vortex position in our experiment suggests new applications. For instance, the possibility of handling objects inside a drop or in the surrounding fluid can be hoped in a way similar to the one recently demonstrated by Chung and Cho [13].

\section{EWOD chip technology}

\subsection{Microfabrication}

The EWOD chip is microfabricated from a $200 \mathrm{~mm}$ silicon wafer. The fabrication process is based on conventional techniques of microelectronics. The lab-on-chip shown in Fig. 1 is entirely realized in the CEA-LETI-MINATEC clean rooms. The process flow used

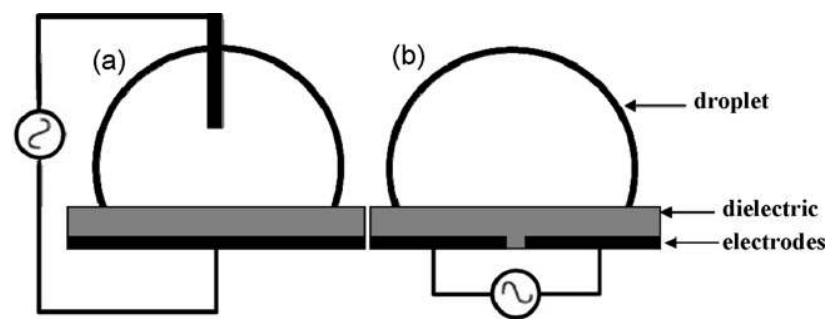

Fig. 2. (a) 'Classical' configuration: a needle electrode is immersed into the droplet. (b) Coplanar electrode configuration: the droplet straddles the two electrodes. is outlined in Fig. 3(a-f). The first step is a $1 \mu \mathrm{m}$ thick silicon oxide layer obtained by a wet thermal oxidation (a). Then a first $200 \mathrm{~nm}$ thick AlSi metal layer is deposited (b). This step is followed by a photolithography and a dry etching step to pattern the electrical connection lines $(20 \mu \mathrm{m}$ width). A $300 \mathrm{~nm}$ thick dielectric layer of $\mathrm{Si}_{3} \mathrm{~N}_{4}$ is then deposited using Plasma Enhanced Chemical Vapour Deposition (PECVD) (c). Tiny holes (15 $\mu \mathrm{m} \times 15 \mu \mathrm{m})$ are photolithographied and etched in order to enable the connection between the first and the second metal layers obtained by the same process (200 nm thick AlSi) (d). This second metal layer is devoted to the electrode patterns. The use of two metal layers gives more flexibility in the design of the chips. Electrical line connection crossings are avoided and complex electrode areas can be patterned. Another PECVD step enables the covering of these electrodes with a $600 \mathrm{~nm}$ thick dielectric layer of $\mathrm{Si}_{3} \mathrm{~N}_{4}$ (e). The electrical permittivity $\varepsilon$ and breakdown strength of this layer are respectively $6.3 \varepsilon_{0}$ and $1 \mathrm{kV} \mu \mathrm{m}^{-1}$. This insulating layer is opened by a photolithography and a dry etching to enable the electrical connection of the chip to the voltage generator. The $22 \mathrm{~mm} \times 22 \mathrm{~mm}$ chips are then individualized and covered with a $1 \mu \mathrm{m}$ thick SiOC hydrophobic layer $\left(\varepsilon=2.75 \varepsilon_{0}\right)$ which is a standard material used in CMOS technology. This SiOC (organosiloxane molecule) layer is deposited using PECVD (f). Contrary to Teflon ${ }^{\circledR}$, SiOC is compatible with the process used in clean rooms. A large range of thickness is allowed from a few $\mathrm{nm}$ to several $\mu \mathrm{m}$ with good homogeneity and robustness [14]. As a surface layer, it must be chemically compatible with the use of biological samples and its properties must be reproducible in terms of contact angle and hysteresis. A low contact angle hysteresis and a high hydrophobic surface facilitate the displacement of the liquid droplet. Careful attention has thus been brought to the choice of the precursor and plasma conditions enabling interesting wetting properties: the zero contact angle between a water droplet and the air is $105^{\circ}, 165^{\circ}$ between a water droplet and silicone oil while the contact angle hysteresis is less than $10^{\circ}$ in both cases.

In order to benefit from this stabilised EWOD technology, the same process flow is used for the present chip. The simple chip design is shown in Fig. 3(g); two half disk shaped coplanar electrodes ( $2 \mathrm{~mm}$ radius) are separated by a $100 \mu \mathrm{m}$ gap. The electrode size allows experiments to be performed with droplets in the $\mu \mathrm{l}$ range. This droplet size is typical of volume reactions used in labon-chips, and can be easily visualized with standard optic devices.

\subsection{EWOD validation}

This section aims to characterise the electrowetting behaviour of the droplet in the coplanar electrode configuration, to evaluate 
(a)

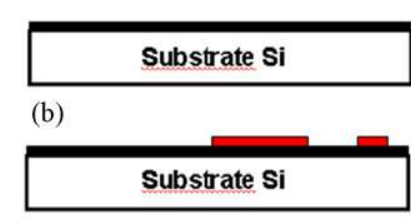

(c)
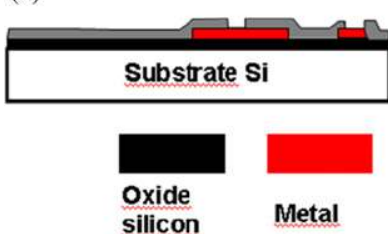

(d)

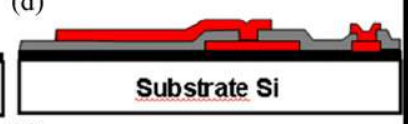

(e)

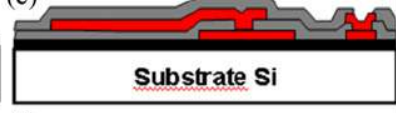

(f)

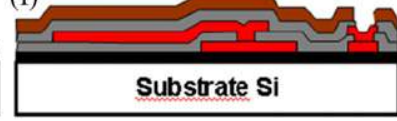

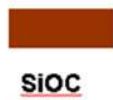

(g)

Fig. 3. (a-f) Process flow; (g) coplanar electrode configuration used for experiment. The vertical lines represent the connection lines.

the accuracy of the experimental setup and to validate the piling up technology of the chips. To do so, the contact angle evolution of the droplet when electric voltage is applied is compared to theoretical curves.

Considering the droplet as a perfect conductor (hypothesis discussed in Section 5.2) the governing equation for EWOD in the 'classical' configuration is the Lippmann-Young equation:

$\cos \left(\theta_{\mathrm{V}}\right)=\cos \left(\theta_{0}\right)+\frac{C V^{2}}{2 \gamma}$

where $\theta_{0}$ is the zero voltage contact angle, $\theta_{\mathrm{V}}$ is the contact angle modified by the electrical potential $V$, which in the case of AC voltage is the root mean square (RMS) voltage, $C$ is the capacitance value per surface unit of droplet-solid interface and $\gamma$ is the surface tension between the droplet and the surrounding oil.

On the coplanar electrode configuration, the droplet straddles the two electrodes. Derived from the calculation presented in [15], and taking into account the double insulating layers (dielectric layer: $\mathrm{Si}_{3} \mathrm{~N}_{4}$, hydrophobic layer: $\left.\mathrm{SiOC}\right), \mathrm{Eq}$. (1) finally reads:

$\cos \left(\theta_{\mathrm{V}}\right)=\cos \left(\theta_{0}\right)+\frac{\varepsilon_{1} \varepsilon_{2} V^{2}}{\left(\left(\varepsilon_{2} d_{1}+\varepsilon_{1} d_{2}\right) 8 \gamma\right)}$

where the subscripts 1 and 2 indicate the $\mathrm{Si}_{3} \mathrm{~N}_{4}$ insulating layer and SiOC hydrophobic layer respectively, $\varepsilon$ is the electrical permittivity and $d$ is the layer thickness.

The experimental protocol of the EWOD validation experiment is similar to the one used in [16]. A picture of the droplet is taken at different values of the applied voltage. The optical axis of the camera is parallel to the electrode gap. After each picture acquisition, the voltage is set to zero and then incremented. The frequency of the applied voltage is fixed to $3 \mathrm{kHz}$ and the $1.5 \mu \mathrm{l}$ droplet solution, composed of a standard Tri-EDTA buffer is inserted into silicon oil. The surface tension between the drop and the surrounding oil is $28 \mathrm{mN} / \mathrm{m}$ and the electrical conductivity of the droplet is $880 \mu \mathrm{S} / \mathrm{cm}$. The voltage range is between 0 Vrms and 92 Vrms. A Matlab program using GUI and the image processing toolbox has been developed to automatically analyse the picture sequence, measure contact angles and plot the electrowetting curves.

As shown from Fig. 4 the experimental data is very close to the curve provided by the theory (Eq. (2)). A maximal discrepancy of $2^{\circ}$ is observed which compares favourably to the typical measurement uncertainty $\left(4^{\circ}\right)$. The droplet presents a highly non-wetting configuration $\left(165^{\circ}\right)$ at zero voltage and the contact angle decreases to a value of about $100^{\circ}$ at $90 \mathrm{~V}$. This corresponds to the contact angle obtained from half the voltage applied with the 'classical' configuration.
As shown in the curves, the contact angle saturation is never reached. This ensures that our experiments remain in the theoretically predicted domain of electrowetting and lowers the risks of trapping charge, of surface deterioration or charge injection within the drop, these last parasitic phenomena being able to generate physical mechanisms that are far beyond the scope of this study.

In conclusion, classical EWOD experiments have been performed at $3 \mathrm{kHz}$. The electrowetting of the droplet matches the theory well which allows us to go forward with the experiments in all confidence. The next step consists in varying the input frequency, observing the hydrodynamic flows within the droplet and the instantaneous surface droplet shape.

\section{Experimental procedure and results}

The chip is immersed in silicone oil (Fig. 5(a)). A diagram of the experimental setup is shown in Fig. 5(b). A $1.5 \mu$ l droplet of Phosphate Buffered Saline (PBS) is placed on the chip surface with a micropipette. The droplet solution contains $10 \mu \mathrm{m}$ diameter fluorescent beads (Intertek CTMA, Duke Scientific) and some additional surfactants. The beads, used as tracers, have a density of $1.05 \mathrm{~g} / \mathrm{cm}^{3}$. The surface tension between the silicone oil and the droplet is $21 \mathrm{mN} / \mathrm{m}$. Due to the small volume of liquid available for the experiments, it was not possible to measure the electrical conductivity. The AC electrical signal was generated using a generator (Yokogawa) and a home-made amplifier. The amplitude of the applied AC voltage, $\mathrm{V}$, was $90 \mathrm{Vrms}$ and the frequency, $f$, ranged from $150 \mathrm{~Hz}$ to $3 \mathrm{kHz}$. A CCD camera (Pixelfly) and a zoom lens were used for image acquisition. Droplet oscillations were visualised using stroboscopic lighting produced by a standard LED. The LED is plugged into the AC generator so that the stroboscopic frequency can be synchronised with the applied voltage. By shifting the phase between AC actuation and stroboscopic lighting, the instantaneous shape of the droplet can be observed during one period of oscillation.

The fluorescent beads are excited by a $470 \mathrm{~nm}$ blue light produced by a mercury lump and a blue filter. A $535 \mathrm{~nm}$ filter is fitted to the zoom lens to detect fluorescence. It is thus possible to characterise simultaneously both the flow and the droplet oscillations at different frequencies of the applied voltage. With the beads and the lighting source used in experiment, the flow that is observed is the droplet surface flow; for the observation of internal flows, the use of a laser sheet would be required.

\subsection{Vortex flow}

Fig. 6(a) corresponds to the side view of the flow along the droplet surface for an input frequency: $f=150 \mathrm{~Hz}$. At the beginning 


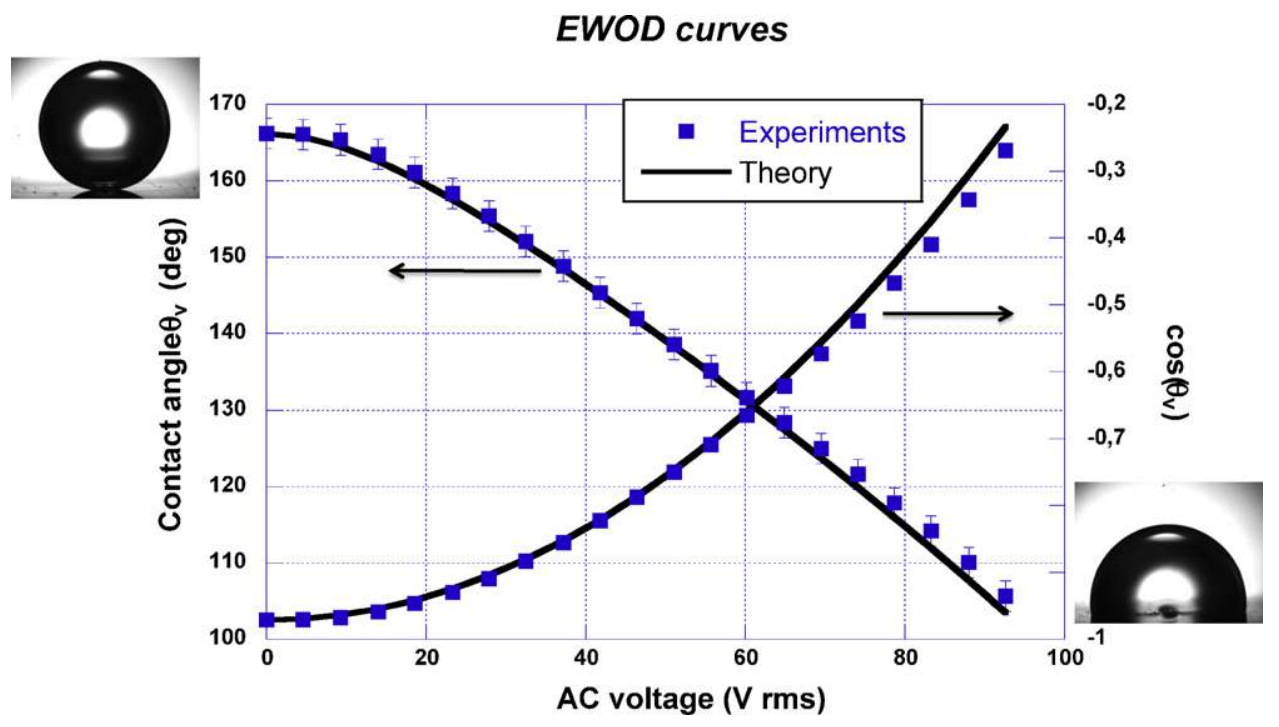

Fig. 4. Electrowetting curves in the coplanar electrode configuration.
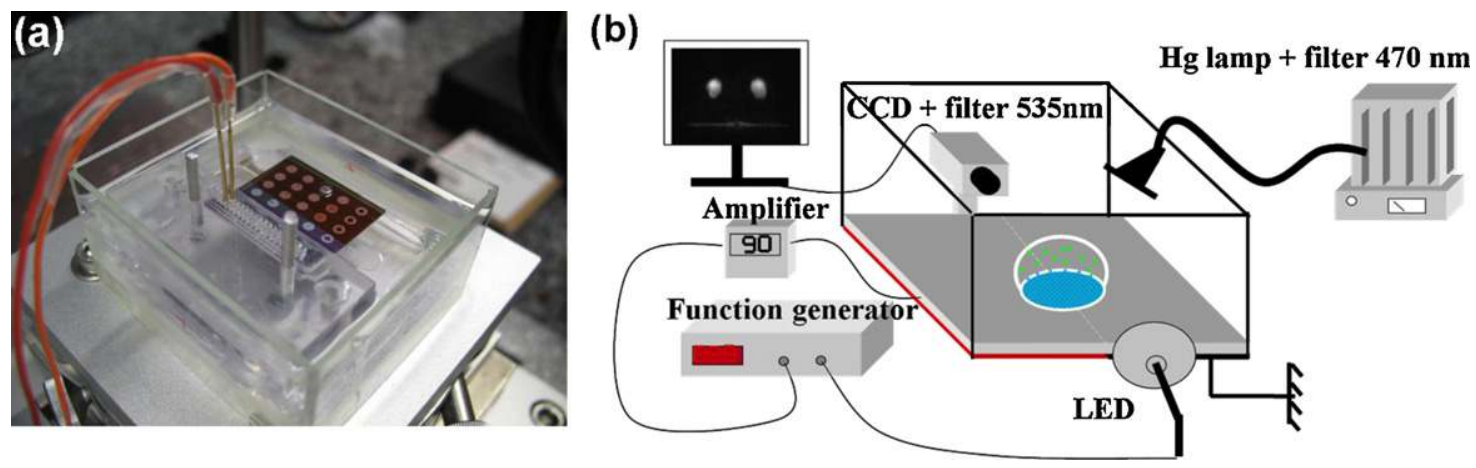

Fig. 5. (a) EWOD chip immersed in silicone oil. (b) Diagram of the experiment setup.

of the experiment, the beads are dispersed. Owing to the mass density of the tracers, some beads drop onto the chip while others are carried by the flow. Time acquisition of the camera is increased so as to visualize the streamline pattern. The two white spots on the image are the results of an artefact of visualisation, and the streamlines deduced from these experiments are shown in Fig. 6(b). The flow is characterised by a quadripolar structure of four vortices. This flow is permanent and the mean velocity is estimated to be around $1 \mathrm{~mm} / \mathrm{s}$.

By increasing the frequency up to $3 \mathrm{kHz}$, the beads are observed to converge near the triple contact line (TCL) and form clusters at the centre of the vortices where local pressure is the smallest. Once they converge, they remain agglomerated until the end of the experiment. Other tracers (hollow glass beads with a density of $0.3 \mathrm{~g} / \mathrm{cm}^{3}$ ) have been used and demonstrate a similar behaviour.

The position of the four vortices on the droplet surface is steady and, interestingly, when input frequency is changed, the four vortices move to a new position along the droplet surface. Fig. 7(a) shows the change in position of the vortices in side-view images for three different frequencies. Then the camera is displaced to get a top view of the phenomenon (Fig. 7(b)). The range of frequencies varies
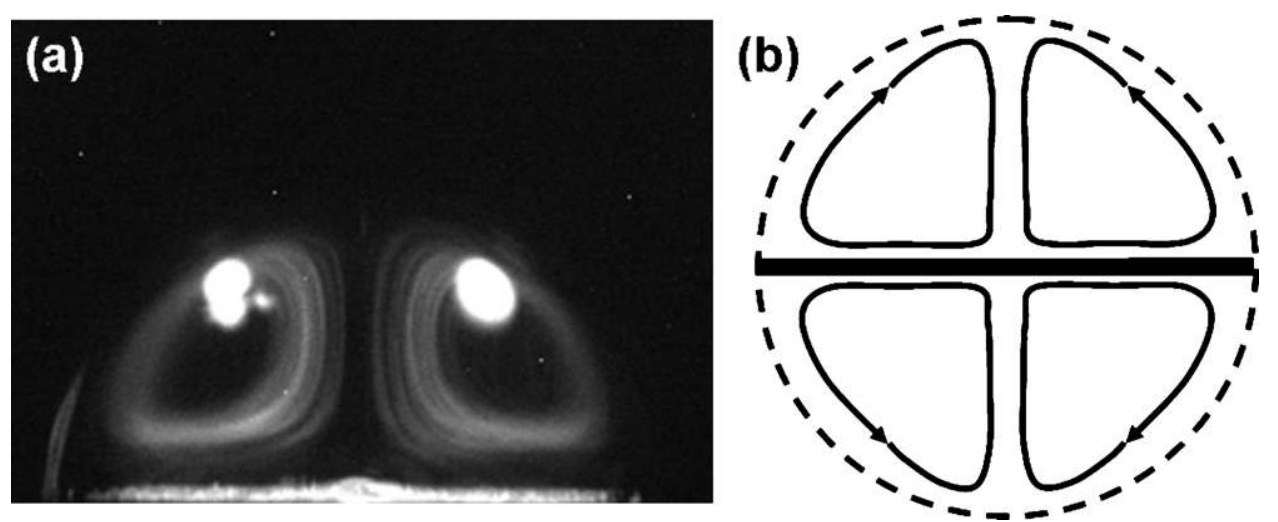

Fig. 6. (a) Side view of the droplet, at an input frequency $f=150 \mathrm{~Hz}$; (b) diagram of the streamlines, the horizontal black line represents the electrode gap. 


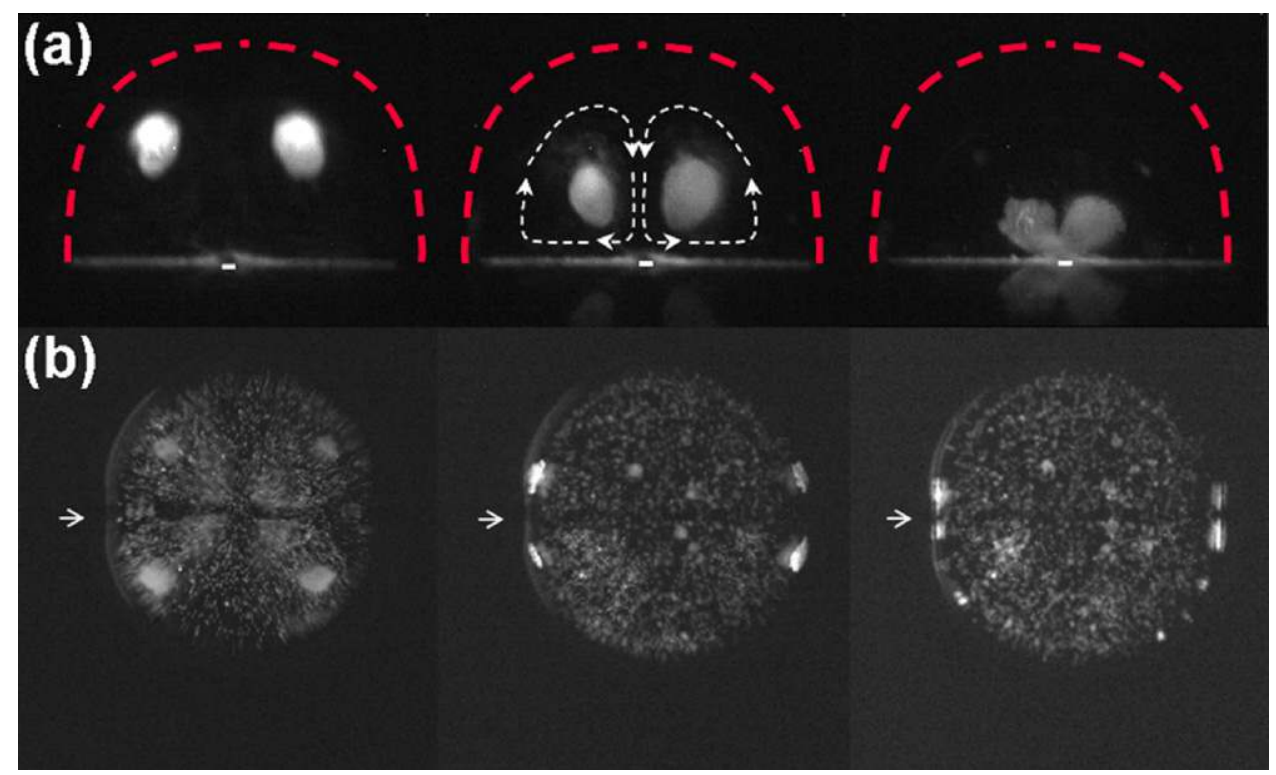

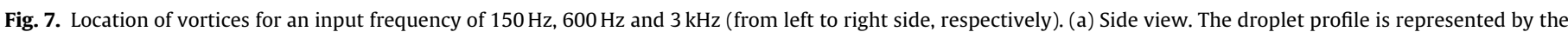
red dashed-line and the electrode gap is represented by the horizontal white segment. (b) Top view, the side view is indicated by white arrows.

from $150 \mathrm{~Hz}$ to $3 \mathrm{kHz}$. The two vortices in the side view and top view images are always symmetrical about the axis of the electrode gap. At a frequency of $3 \mathrm{kHz}$ the vortices are located in the vicinity of the TCL near the electrode gap while almost no motion is observed on the rest of the droplet surface. At a frequency of $150 \mathrm{~Hz}$, the vortices move to mid-height of the droplet and move further from the electrode gap, while at $600 \mathrm{~Hz}$ they stabilise at an intermediate position as illustrated in a supporting movie (referred to as A on the website). This frequency cycle is repeated many times always with the same behaviour: the vortex structures get closer to the electrode gap when the input frequency is increased and they move away from the gap when the applied frequency is decreased. For a given time aperture of the CCD camera, if the voltage frequency is tuned from a low frequency $(150 \mathrm{~Hz})$ to a large frequency $(3000 \mathrm{~Hz})$, it can be checked from videos (see movie A for instance) that the tracers become increasingly distinguishable. This suggests that the typical angular velocity decreases when voltage frequency increases. This point needs to be further investigated with an accurate velocity measurement method (Particle Image Velocimetry or Particle Tracking Velocimetry) adapted to a half-spherical configuration.

These experiments demonstrate a surprising reproducibility and reversibility of vortex locations when actuation frequency ranges from $3 \mathrm{kHz}$ to $150 \mathrm{~Hz}$ and vice versa.

\subsection{Droplet oscillations}

The droplet surface seems to be static when observed with a classical lighting source but in reality, by shifting to stroboscopic lighting, one can observe large droplet surface oscillations induced by AC EWOD (as also supported by companion movies referred to as B-a), B-b) and B-c) on the website). These oscillations are created by the time dependant part of the normal electrical stress acting at the TCL when AC voltage is applied. The electrical stress is proportional to the square of the applied voltage, the frequency of the oscillating stress must thus be twice the input electrical frequency. In our experimental setup, the stroboscopic lighting enables the determination of the oscillation frequency of the droplet and confirms that it oscillates at exactly twice the input frequency. The change in input frequency leads to an apparent change in the droplet shape. By synchronizing the droplet oscillations and the stroboscopic lighting and varying the phase lag between the two electrical signals, an image of the instantaneous shape of the droplet can be obtained (Fig. 8(a)). Image analysis is then possible using the image processing toolbox of Matlab. For each frequency, two droplet images, corresponding to a phase lag between the stroboscopic frequency and the actuation frequency of $0^{\circ}$ and $90^{\circ}$, are analysed. These two images correspond to the extreme oscillating shape of the droplet during a period of oscillation. The droplet radius is plotted in Fig. 8 (b) (blue curve for the $0^{\circ}$ phase lag, black curve for $90^{\circ}$ phase lag).

The plots show that the amplitude of the droplet oscillations decreases as the frequency of the input voltage increases. For $f=150 \mathrm{~Hz}$, the amplitude of the oscillations at the apex of the droplet is $69 \mu \mathrm{m}$ while for a frequency $f=600 \mathrm{~Hz}$, the oscillations are damped and the oscillation amplitude at the apex of the droplet hardly reaches $10 \mu \mathrm{m}$. At $3 \mathrm{kHz}$, this amplitude is only $3 \mu \mathrm{m}$. The number of nodes and troughs seems to increase as the frequency increases, revealing a change in the droplet mode oscillations. This is visible on the curves corresponding to the frequencies of $150 \mathrm{~Hz}$ and $300 \mathrm{~Hz}$, and one can recognize respectively the eighth and twelfth eigenmode. Beyond $600 \mathrm{~Hz}$, the amplitude of the oscillations is so small that distinction between nodes and troughs becomes difficult.

These oscillations also affect the wetting angle. At $150 \mathrm{~Hz}$, the contact angle oscillates between $70^{\circ}$ and $95^{\circ}$ while at $300 \mathrm{~Hz}$, the range of oscillation is reduced from $75^{\circ}$ to $90^{\circ}$. At $3 \mathrm{kHz}$, the average wetting angle is $83^{\circ}$ and the difference between the two limit values of the contact angle is only $3^{\circ}$, which is comparable with the measurement error $\left(4^{\circ}\right)$. The onset of detection for the contact angle variation is reached and beyond this frequency, the oscillations are strongly damped: the higher the frequency, the smaller the range of values swept by the oscillating contact angle.

\section{Discussion}

Reproducibility of the vortex flow and droplet oscillations was achieved by using oil as ambient phase, thereby allowing the drop to keep a constant volume by preventing it from evaporating, and also reducing surface hysteresis by enhancing TCL sliding. The SiOC 

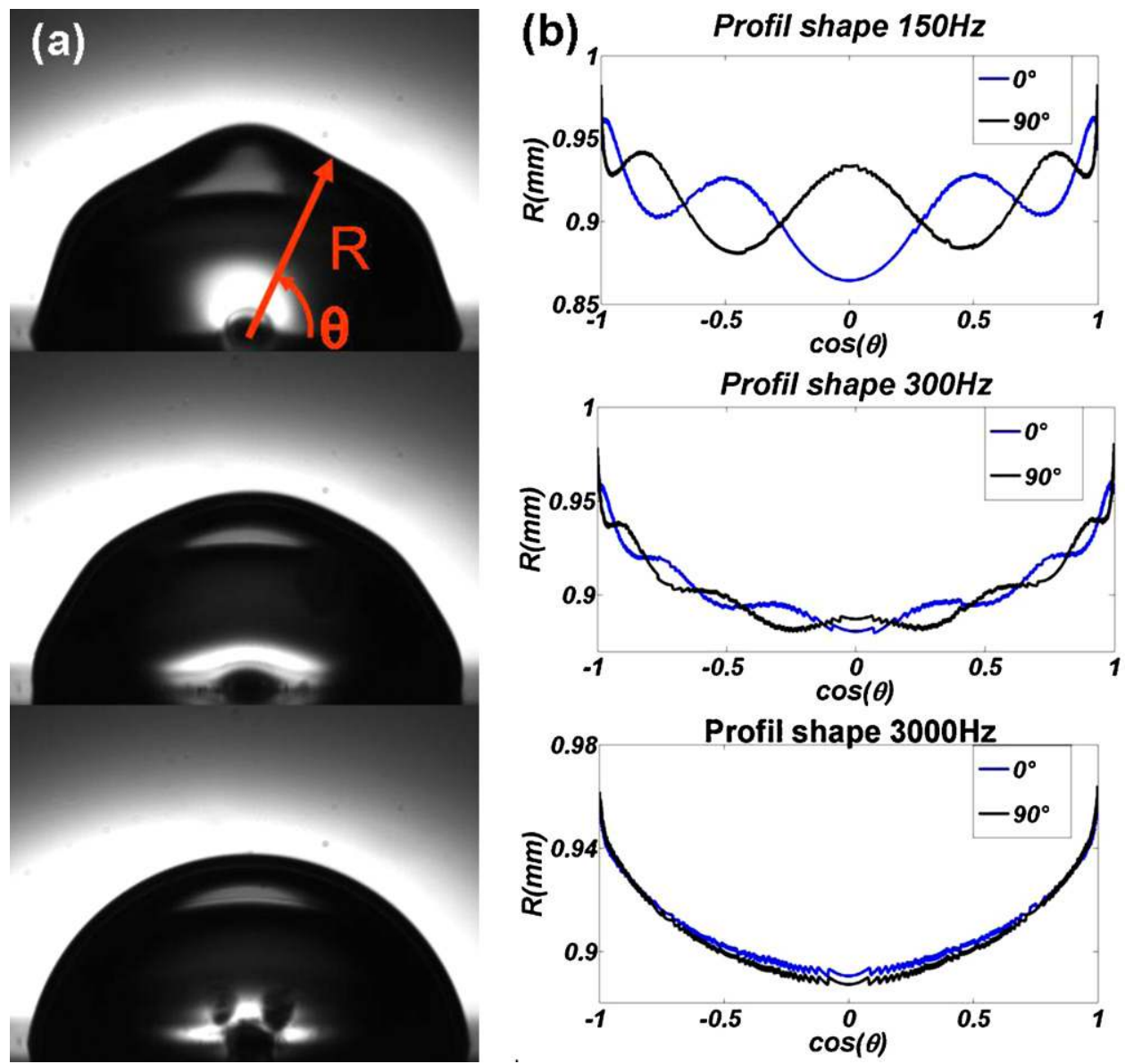

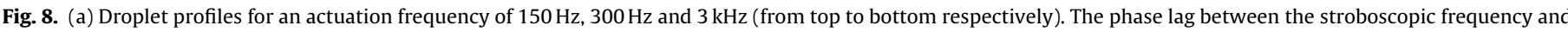

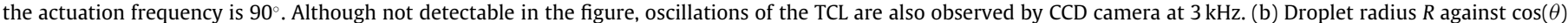

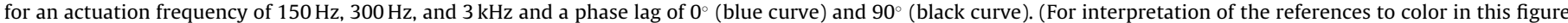
legend, the reader is referred to the web version of the article.)

coating also allows us to achieve sensitive and reproducible electrowetting experiments.

A correlation between droplet oscillation and induced hydrodynamic flows was earlier reported by Ko et al. [17] In the present experiment there may also be a strong link between oscillation mode and vortex flow (Fig. 9). More precisely, the vortices may be located at a latitude where surface deformation is the largest. The change of the input frequency leads to a change of oscillation modes and consequently a change of vortex location.

However, the four symmetrical flow patterns remain intriguing. Contrary to the 'classical' configuration, the coplanar electrode configuration is not 2-D axisymmetrical. According to the flow patterns we observe, it seems obvious that the electrode gap plays a significant role in the four vortex structure. Some tangential stress may arise from the electrode gap. Two interpretations for such a quadripolar structure can thus be proposed:

\subsection{Non axisymmetrical Maxwell normal electrical stress inducing azimuthal oscillation modes}

EWOD is commonly attributed to the action of the normal component of the Maxwell stress, $(\boldsymbol{T e} \cdot \boldsymbol{n}) \cdot \boldsymbol{n}$ with $\boldsymbol{n}$ the outward unit

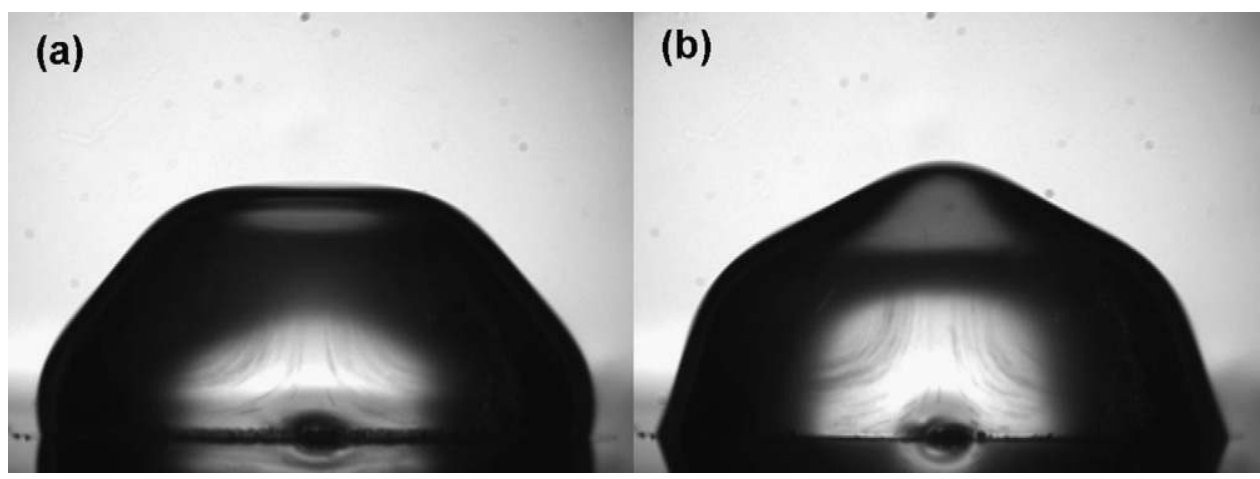

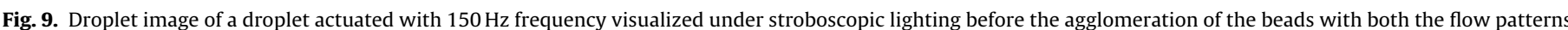
and the oscillating shape: (a) phase lag is $0^{\circ}$; (b) phase lag is $90^{\circ}$. 
vector at the TCL. When the voltage is switched on, surface electrical charges Qs concentrate along the TCL, according to the Gauss' law which reads, at the droplet surface:

$Q s=\|\varepsilon \boldsymbol{E} \cdot \boldsymbol{n}\|$

where $\|a \cdot \boldsymbol{n}\|$ denotes the jump of a quantity $a$ across a dividing interface, $\boldsymbol{E}$ is the electric field vector and $\boldsymbol{n}$ is the outward unit normal vector.

These electrical charges submitted to the normal component of the electric field give rise to the normal component of the Maxwell electrical stress which pulls the TCL, and makes the droplet to wet. A first hypothesis that could explain the four vortex structure is that the presence of the electrode gap $(100 \mu \mathrm{m})$ may locally disturb the normal electrostatic stress at the vicinity of the TCL such as:

$\frac{\partial((\boldsymbol{T e} \cdot \boldsymbol{n}) \cdot \boldsymbol{n})}{\partial s} \neq 0$

where $s$ is the curvilinear abscissa along the TCL. Eq. (4) can induce a non-perfect axisymmetrical shape of the droplet oscillations with azimuthal oscillation modes. A careful observation of the top view of the droplet near the electrode gap (Fig. 7) shows that the mean droplet shape is not perfectly circular and that a default of circularity may occur at the electrode gap as represented in Fig. 10. Further investigation on the droplet shape at the vicinity of the electrode gap has to be carried out. If this point is confirmed, oscillations based on this particular droplet shape may cause the typical flow pattern.

\subsection{Maxwell tangential electrical stress}

As suggested in [18], additional driven mechanisms for internal flows have to be investigated. We discuss here the existence of the Maxwell tangential electrical stress at the surface of the droplet located at the vicinity of the electrode gap (in or near the wetting plane).

In EWOD modelling, droplets have been considered for a long time as perfect electrical conductors. This (simplifying) hypothesis implies that the electric field is exclusively normal to the droplet surface, which means that no tangential electric field can exist. Yet, in our case, a local investigation of the electrostatics near the electrode gap proves to be necessary.

In the bulk, although the electrical conductivity of the droplet solution has not been measured due to the small volume of liquid, its electrical conductivity is estimated to be close to that of PBS solution which was measured as $\sigma=1.6 \mathrm{~S} / \mathrm{m}$. With such an electrical conductivity, the relaxation time $\left(\varepsilon / \sigma \approx 10^{-10} \mathrm{~s}\right)$ is so small compared to the electrical time scale $\left(1 / f \approx 10^{-4} \mathrm{~s}\right)$ that the bulk can be

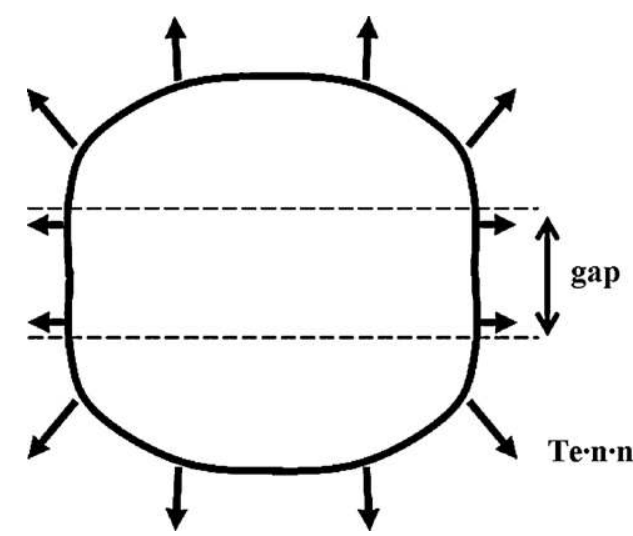

Fig. 10. Diagram of a possible distribution of the normal component of the Maxwell electrical stress Te.n.n. Non-axisymmetrical oscillation modes could result and generate a four vortex flow structure.

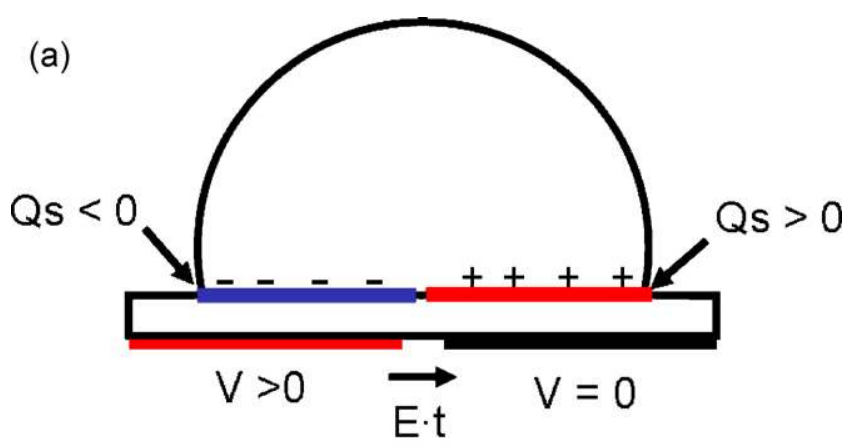

(b)

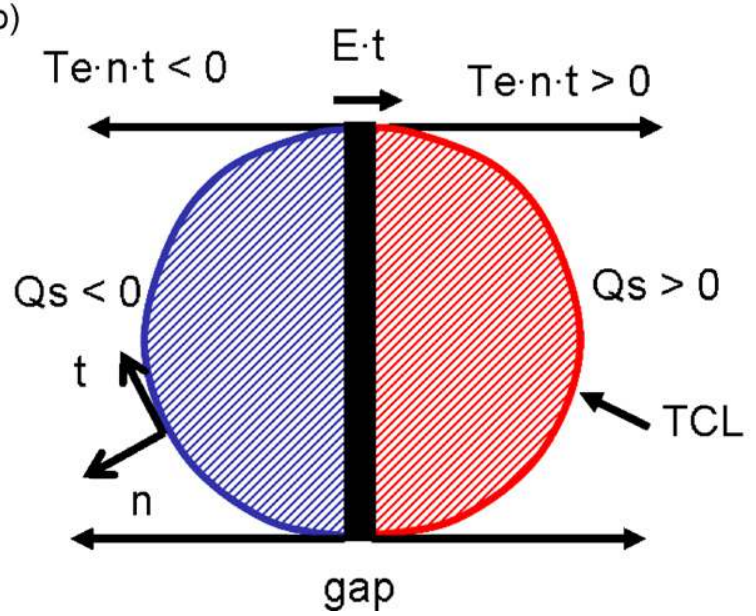

Fig. 11. Schematic of the tangential component of the Maxwell electrical stress, Te.n.t. (a) Representation of the electric surface charge on both side of the electrode gap. (b) As a result, opposite tangential stresses that could explain the flow patterning.

considered as free of volumic charge in our experiments. The only non-vanishing electrical charge accumulates along the drop surface, especially at/near the TCL the electrode gap where a tangential component of the electrical field $\boldsymbol{E} \cdot \boldsymbol{t}$, with $\boldsymbol{t}$ a unit vector along the $\mathrm{TCL}$, is expected. The joint contribution of the surface charge and the tangential electric field gives rise to a Maxwell stress Te.n.t that is balanced by a viscous stress to produce the hydrodynamic flow [19]:

$\|(\boldsymbol{T e}+\boldsymbol{T m}) \cdot \boldsymbol{n}\| \cdot \boldsymbol{t}=0$

where $\boldsymbol{T} \boldsymbol{m}$ is the viscous stress tensor, $\boldsymbol{n}$ and $\boldsymbol{t}$ are the normal and tangential unit vector at/along the TCL respectively. Above the right electrode (Fig. 11(a)), positive surface charge accumulates at the liquid-solid interface and particularly at the TCL. Above the counter electrode, the sign of the surface charge is opposite. The direction of the tangential electric field (Fig. 11(b)) remains constant which would create a pair of two opposite Maxwell tangential stress on either side of the electrode gap. This stress together with the oscillations, may contribute to this flow pattern. A study of the dependence of the four vortex structure flow on the electrical conductivity of the droplet as well as a calculation of the Maxwell stress has to be achieved in order to estimate the tangential electrical stress in this configuration. Owing to the singularity of the electric field at the TCL, a non-trivial model is required.

\section{Conclusion}

An electrohydrodynamic phenomenon has been studied in the coplanar electrode configuration. The electrowetting of a droplet on such a configuration has been validated as well as the reliability of the SiOC coating. A new surface flow induced by AC EWOD has 
been analysed. A perfectly quadripolar flow with four symmetrical vortex structures has been demonstrated. Droplet oscillations are probably involved in this hydrodynamic flow but the source of the typical flow pattern still remains questionable. Two possible driven mechanisms are proposed; the relevancy of new theoretical developments is made evident but this goes beyond the scope of this paper. Potential applications of this research include, for example, the handling of cells or macromolecules with biological sample preparation. The ability to control the vortex position precisely by adjusting only one input frequency is a key factor for promoting the use of such EWOD induced flows in future lab-on-chips.

\section{Appendix A. Supplementary data}

Supplementary data associated with this article can be found, in the online version, at doi:10.1016/j.snb.2009.12.066.

\section{References}

[1] A.L. Yarin, W. Liu, D.H. Reneker, Motion of droplets along thin fibers with temperature gradient, J. Appl. Phys. 91 (7) (2002) 4751-4760.

[2] A. Buguin, L. Talini, P. Silberzan, Ratchet-like topological structures for the control of micro-drops, Appl. Phys. A 75 (2002) 207-212.

[3] Y. Fouillet, D. Jary, C. Chabrol, P. Claustre, C. Peponnet, Digital microfluidic design and optimization of classic and new fluidic functions for lab on chip systems, Microfluid. Nanofluid. 4 (2008) 159-165.

[4] R. Sista, Z Hua, P. Thwar A. Sudarsan, V. Srinivasan, A. Eckhardt, M. Pollack V. Pamula, Development of a digital microfluidic platform for point of care testing, Lab Chip 8 (2008) 2091-2104.

[5] A.R. Wheeler, H. Moon, C.A. Bird, R.R. Ogorzalek Loo, C.J. Kim, J.A. Loo, R.L. Garrell, Digital microfluidics with in-line sample purification for proteomics analyses with MALDI-MS, Anal. Chem. 77 (2005) 534-540.

[6] D. Jary, A. Chollat-Namy, Y. Fouillet, J. Boutet, C. Chabrol, G. Castellan, D. Gasparutto, C. Pepponet, DNA repair enzyme analysis on EWOD fluidic microprocessor, NSTI Nanotech, 2006 Technical Proceedings, vol. 2, pp. 554-557.

[7] S.H. Ko, H. Lee, K.H. Kang, Hydrodynamic flows in electrowetting, Langmuir 24 (2008) 1094-1101

[8] T.B. Jones, M. Gunji, M. Washizu, M.J. Feldman, Dielectrophoretic liquid actuation and nanodroplet formation, J. Appl. Phys. 89 (No 3) (2001).

[9] P. Sen, C.J. Kim, Capillary spreading dynamics of electrowetted sessile droplets in air, Langmuir 25 (8) (2009) 4302-4305.
[10] M. Abdelgawad, S.L.S. Freire, H. Yang, A.R. Wheeler, All terrain droplet actuation, Lab. Chip 8 (2008) 672-677.

[11] R. Miraghaie, J.D. Sterling, A. Nadim, Shape oscillation and internal mixing in sessile liquid drops using electrowetting-on-dielectric(EWOD), NSTI-Nanotech 2 (2006) 610-613.

[12] F. Mugele, J.C. Baret, D. Steinhauser, Microfluidic mixing through electrowetting-induced droplet oscillations, Appl. Phys. Lett. (2006) 88

[13] S.K. Chung, S.K. Cho, On-chip manipulation of objects using mobile oscillating bubbles, J. Micromech. Microeng. 18 (2008) 125024.

[14] J. Thery, M. Borella, S. Le Vot, D. Jary, F. Rivera, G. Castellan, A.G. Brachet, M. Plissonnier, Y. Fouillet, Sioc as a hydrophobic layer for electrowetting on dielectric applications, Proceedings of $\mu$ Tas 2007, no. 1, pp. 349-351, ISBN: 978-0-9798064r-r0-7.

[15] C.G. Cooney, C.Y. Chen, M.R. Emerling, A. Nadim, J.D. Sterling, Electrowetting droplet microfluidics on a single planar surface, Microfluid. Nanofluid. 2 (2006) 435-446.

[16] S. Berry, J. Kedzierski, B. Abedian, Low voltage electrowetting using thin fluoroploymer films, J. Colloid Interface Sci. 303 (2006) 517-524.

[17] S.H. Ko, S.J. Lee, K.H. Kang, A synthetic jet produced in aqueous solution, Appl Phys. Lett. 94 (2009) 194102.

[18] L. Davoust, Y. Fouillet, Y. Ishida, Drop stirring flow under ewod and ehd actuation: a new step towards biological sample preparation, Proceedings of $\mu$ Tas 2007, (2), pp. 925-927, ISBN: 978-0-9798064r-r0-7.

[19] J.R. Melcher, G.I. Taylor, Electrohydrodynamics: a review of the role of interfacial shear stresses, Ann. Rev. Fluid Mech. 1 (1969) 111.

\section{Biographies}

R. Malk received his Engineer diploma in fluid mechanics at the Institut National Polytechnique de Grenoble (INPG) in 2007. Since 2007, he is working towards a PhD degree at CEA/LETI. His research focuses on hydrodynamic flows induced by Electrowetting on Dielectric.

Y. Fouillet received his "Agrégation de mécanique" diploma in 1987 and a PhD in the numerical simulation of shear flow from the Institut National Polytechnique de Grenoble (INPG) in 1992. In 1992, he joined the CEA/LETI for MEMS development. Since 1998 he has worked on microfluidics. His current research interests include lab-on-chip, droplet handling, electrowetting and flow in microchannels.

L. Davoust received his Engineer diploma and his $\mathrm{PhD}$ in buoyancy-driven MHD flows from the Institut National Polytechnique de Grenoble (INPG) in 1993 and 1996 , respectively. In 1997, he joined CNRS as a permanent researcher (LEGI Laboratory). His current research topics include lab-on-chips, free label biosensors, nanoimprint and flow-induced nanopatterning. 